\title{
A local renal renin-angiotensin system activation via renal uptake of prorenin and angiotensinogen in diabetic rats
}

This article was published in the following Dove Press journal:

Diabetes, Metabolic Syndrome and Obesity: Targets and Therapy 18 January 2016

Number of times this article has been viewed

\author{
Akihiro Tojo' \\ Satoshi Kinugasa' \\ Toshiro Fujita ${ }^{2}$ \\ Christopher SWilcox ${ }^{3}$ \\ 'Division of Nephrology and \\ Endocrinology, ${ }^{2}$ Research Center for \\ Advanced Science and Technology, \\ The University of Tokyo, Tokyo, \\ Japan; ${ }^{3}$ Division of Nephrology \\ and Hypertension, Center for \\ Hypertension, Kidney and Vascular \\ Research, Georgetown University, \\ Washington, DC, USA
}

\begin{abstract}
The mechanism of activation of local renal renin-angiotensin system (RAS) has not been clarified in diabetes mellitus (DM). We hypothesized that the local renal RAS will be activated via increased glomerular filtration and tubular uptake of prorenin and angiotensinogen in diabetic kidney with microalbuminuria. Streptozotocin (STZ)-induced DM and control rats were injected with human prorenin and subsequently with human angiotensinogen. Human prorenin uptake was increased in podocytes, proximal tubules, macula densa, and cortical collecting ducts of DM rats where prorenin receptor (PRR) was expressed. Co-immunoprecipitation of kidney homogenates in DM rats revealed binding of human prorenin to the PRR and to megalin. The renal uptake of human angiotensinogen was increased in DM rats at the same nephron sites as prorenin. Angiotensin-converting enzyme was increased in podocytes, but decreased in the proximal tubules in DM rats, which may have contributed to unchanged renal levels of angiotensin despite increased angiotensinogen. The systolic blood pressure increased more after the injection of $20 \mu \mathrm{g}$ of angiotensinogen in DM rats than in controls, accompanied by an increased uptake of human angiotensinogen in the vascular endothelium. In conclusion, endocytic uptake of prorenin and angiotensinogen in the kidney and vasculature in DM rats was contributed to increased tissue RAS and their pressor response to angiotensinogen.
\end{abstract}

Keywords: renal renin-angiotensin system, prorenin, angiotensinogen, diabetic nephropathy, microalbuminuria

\section{Introduction}

The renin-angiotensin system (RAS) was originally considered to be a circulating system that regulated sodium and fluid balance and hemodynamics. However, more recent studies have detected a local RAS in the kidneys that was independent of the systemic RAS. ${ }^{1-4}$ Whereas the systemic RAS activity was reported to be normal or suppressed in diabetes mellitus (DM), ${ }^{5-7}$ most studies have reported that the local renal RAS was upregulated, ${ }^{5,8-11}$ but the mechanisms underlying these findings have not been clarified. This is important because RAS inhibition is a mainstay of treatment for proteinuric diabetic nephropathy.

Activation of the classical circulating RAS is initiated by the release of renin and prorenin from juxtaglomerular cells of the kidney. ${ }^{12,13}$ Circulating prorenin can be extracted by organs, including the kidney and converted locally to renin by binding to the prorenin receptor (PRR). ${ }^{13}$

The messenger RNA (mRNA) and protein of all of the components of the RAS have been detected in the kidney where they might function as an endogenous renal RAS. ${ }^{3,8,14,15}$ However, since cells expressing angiotensinogen mRNA may not produce
Correspondence: Akihiro Tojo Division of Nephrology and Endocrinology, The University of Tokyo, 7-3-I Hongo, Bunkyo-ku, Tokyo II 3-8655, Japan

Tel +8I $3381554 I I$ ext 37219

$\mathrm{Fax}+81338140021$

Email akitojo-tky@umin.ac.jp (c) (i) (5) 2016 Tojo et al. This work is published and licensed by Dove Medical Press Limited. The full terms of this license are available at https://www.dovepress.com/terms.php

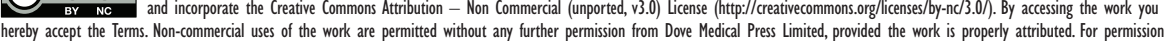
hereby accept the Terms. Non-commercial uses of the work are permitted without any further permission from Dove 
angiotensinogen protein, ${ }^{16}$ it is possible that components of the renal tissue RAS are derived from distant organs, filtered by the glomerulus, and reabsorbed by the renal tubules. Indeed, although the mRNA for angiotensinogen was readily detected in proximal tubular cells of mice, nearly all of the proximal tubular angiotensinogen protein originated in the liver. ${ }^{17}$

An impaired glomerular filtration barrier in diabetic nephropathy will increase the filtration of some RAS components whose molecular sizes are smaller than albumin (68 kDa), including prorenin $(47 \mathrm{kDa})$ and angiotensinogen $(52 \mathrm{kDa})$. Whereas, megalin mediates the endocytosis of angiotensinogen by the proximal tubule, ${ }^{16}$ the PRR is expressed on podocytes, intercalated cells of the cortical collecting duct (CCD), and vascular smooth muscle cells and could mediate prorenin endocytosis. ${ }^{18}$

In the present study, we examined the hypothesis that circulating human angiotensinogen and human prorenin are extracted by the kidney and the vascular endothelium in the diabetic rat and contribute to a local renal and vessel wall RAS and to a pressor system.

\section{Materials and methods}

\section{Animals and experimental designs}

Male Sprague Dawley rats (Charles River Laboratories, Shizuoka, Japan) weighing 130-150 g were housed in a temperature- and humidity-controlled room with access to tap water and standard animal chow. Rats were allocated as controls $(n=6)$ or diabetes $(n=7)$ group. DM was induced by an intravenous (IV) injection of streptozotocin (STZ) (60 mg/kg body weight in citrate buffer) from the tail vein. After 4 weeks, diabetes and microalbuminuria were confirmed by measurement of the urinary glucose and protein by dipstick. Animals were anesthetized with pentobarbital (50 $\mathrm{mg} / \mathrm{kg}$ body weight) prior to invasive procedures. All procedures were conducted in accordance with the Guide for Animal Experimentation of the Faculty of Medicine, The University of Tokyo (Tokyo, Japan) and the National Institutes of Health (NIH) Guide for the Care and Use of Laboratory Animals. This study was approved by the Medical Experimental Animal Ethics Committee of the University of Tokyo (approval number P10-079), and for this study and the use of human prorenin and angiogtensinogen we also followed the principles outlined in the Declaration of Helsinki.

\section{Administration of human prorenin and angiotensinogen}

Rats were anesthetized for IV injections of $20 \mu \mathrm{g}$ recombinant human prorenin (Innovative Research Inc., Novi, MI, USA, dissolved in $0.2 \mathrm{~mL}$ saline) through a jugular vein catheter. Subsequently, incremental doses of recombinant human angiotensinogen (Calbiochem, San Diego, CA, USA) were injected at 5-minute intervals $(0.02-20 \mu \mathrm{g} / 0.2 \mathrm{~mL}$ saline). Blood pressure (BP) was recorded from a catheter in the abdominal aorta. Both kidneys were removed 15 minutes after completion of the IV injections. The right kidney was used for renal tissue angiotensin (Ang II) measurement and Western blot analyses, and the left kidney was fixed with periodate-lysine-paraformaldehyde solution, embedded in wax and used for the immunostaining for human prorenin, angiotensinogen, and rat PRR.

\section{Measurement of the renal tissue Ang II and the urinary protein, creatinine, and glucose levels}

The renal tissue Ang II concentration was measured by radioimmunoassay and related to the protein content of the kidney homogenate. ${ }^{19}$ Urine samples were collected in metabolic cages. Protein and creatinine concentrations were measured by spectrophotometry. ${ }^{20}$ Blood glucose concentration was measured by Glutest ProR (Sanwa Kagaku Kenkyusho, Nagoya, Japan) using tail vein blood under the condition with free access to food.

\section{Immunohistochemistry and immunoelectron microscopy for prorenin, the PRR, angiotensinogen, and angiotensin-converting enzyme}

Light microscopic immunohistochemistry was performed as described. ${ }^{21,22}$ Kidney sections $(2 \mu \mathrm{m})$ were incubated with rabbit polyclonal antibodies against human prorenin (1:100 dilution; Serotech, Oxford, UK), PRR (1:100 dilution; Abcam, Cambridge, UK), or human angiotensinogen (1:100 dilution; Abcam) overnight, and thereafter with a horseradish peroxidase (HRP)-conjugated antirabbit IgG secondary antibody (Dako, Glostrup, Denmark) at a 1:50 dilution for 1 hour. HRP signals were developed by diaminobenzidine (DAB, Dojindo Laboratories, Kumamoto, Japan). The immunoreactivity for angiotensin-converting enzyme (ACE) stained with mouse monoclonal antibody for ACE (1:100 dilution, Chemicon International, Temecula, CA, USA) and HRP-conjugated antimouse IgG secondary antibody (Dako) was semiquantified with scores: 0 , no; 1 , weak; 2 , moderate; 3 , strong staining counting 100 glomeruli and proximal tubules in each group. To detect the subcellular localization of human prorenin, pre-embedding immunoelectron microscopy was performed as described previously. ${ }^{23}$ Tissue sections 
(50 $\mu \mathrm{m})$ cut by a vibratome (Dosaka EM, Kyoto, Japan) were incubated overnight with a rabbit antibody against human prorenin (1:100 dilution) followed by an HRP-conjugated antirabbit IgG secondary antibody (1:50 dilution). HRP signals were developed by DAB, postfixed with $2 \%$ osmium solution, and embedded in epoxy resin. Ultrathin sections were examined by a transmission electron microscope (Hitachi H-7000, Tokyo, Japan).

\section{Western blot analyses of kidney homogenates or isolated glomeruli for} human prorenin, angiotensinogen, and PRR Homogenates of whole kidneys were obtained and glomerular and tubular fractions were separated by graded sieving as described previously. ${ }^{21,24}$ Homogenate proteins $(20 \mu \mathrm{g})$ were loaded on $4 / 20 \%$ gels, separated by electrophoresis, the protein bands transferred to polyvinylidene difluoride membranes (Millipore Co, Billerica, MA, USA), incubated with rabbit antihuman prorenin antibody (1:250 dilution), rabbit anti-PRR antibody (1:500 dilution), or rabbit antihuman angiotensinogen antibody (1:500 dilution) as the primary antibody, and with HRP-conjugated antirabbit immunoglobulin (Dako) as the secondary antibody (1:1,000 dilution). The blots were detected by DAB with nickel chloride. As loading control, the membranes were incubated with anti- $\beta$-actin antibody (Abcam, Tokyo, Japan), and processed as mentioned earlier. The area and density of bands for each protein were measured using a NIH image software program. The specificity of the antibody for human angiotensinogen was confirmed by Western blot analysis using increasing amount $(10,100$, $1,000 \mathrm{ng}$ ) of rat angiotensinogen and human angiotensinogen on the membrane.

\section{Immunoprecipitation of human prorenin extracted from glomerular proteins with antibodies against PRR and megalin}

Kidney homogenates from rats injected with human prorenin and angiotensinogen were incubated with protein A-sepharose for 30 minutes to remove nonspecific precipitates, centrifuged at $5,000 \times \mathrm{g}$ for 1 minute and the supernatant was incubated for 2 hours at $4^{\circ} \mathrm{C}$ with the addition of either a rabbit antiPRR antibody (1:500 dilution) or a rabbit antimegalin antibody and $10 \mu \mathrm{L}$ of protein A-sepharose. After five repeated washes by resuspension in $4^{\circ} \mathrm{C}$ lysis buffer and centrifugation at $2,000 \times g$ for 2 minutes, $20 \mu \mathrm{L}$ of sample buffer was added to the precipitates which were boiled for 2 minutes and centrifuged at $15,000 \times g$ for 5 minutes. The supernatants were subjected to sodium dodecyl sulfate polyacrylamide gel electrophoresis, and a Western blot analysis was performed with rabbit antihuman prorenin antibody at a 1:200 dilution, followed by incubation with an HRP-conjugated secondary antibody at a 1:1,000 dilution and DAB staining.

\section{Statistical analyses}

All data were shown as mean \pm standard error of the mean. The mean values were compared between two groups using the unpaired Student's $t$-test. The BP response to the increasing doses of angiotensinogen was analyzed by a repeated-measures analysis of variance. $P$-values $<0.05$ were considered to be significant.

\section{Results}

STZ-induced diabetic rats had increased blood glucose ( $427 \pm 86$ vs $183 \pm 41 \mathrm{mg} / \mathrm{dL}, \mathrm{DM}$ vs control, $P<0.002)$ and proteinuria $(6.5 \pm 1.9$ vs $1.7 \pm 0.5 \mathrm{mg} / \mathrm{d} / 100 \mathrm{~g}$ body weight, $P<0.05$ ), but their systolic and diastolic BPs were similar (systolic BP: $84 \pm 12$ vs $85 \pm 9 \mathrm{mmHg}$, ns; diastolic BP: $55 \pm 11$ vs $53 \pm 15 \mathrm{mmHg}$, ns).

\section{Changes in the systolic and diastolic BP after the administration of human angiotensinogen and human prorenin in control and STZ-induced diabetic rats}

Continuous BP recording in anesthetized DM rats revealed that an IV injection of human prorenin and human angiotensinogen to these rats dose-dependently increased both systolic and diastolic BP (Figure 1). In contrast, similar injections into normal rats did not produce a significant change from baseline.

\section{The uptake of exogenous human}

\section{prorenin is enhanced in diabetic rats}

Immunostaining for human prorenin was increased in the podocytes, proximal tubules, macula densa, and CCDs of DM rats (Figure 2A-D). This was confirmed by Western blot for human prorenin in whole kidney homogenates, which showed almost twice as much human renal prorenin uptake in DM rat kidneys (Figure 2E and F). The expression of prorenin was increased in both glomerular and tubular fractions (Figure 2A-D). There was more human prorenin in glomeruli and tubules from DM rats (Figure 2G). Pre-embedding immunoelectron microscopy localized the human prorenin in DM rats to the vesicles of podocytes and proximal tubular epithelial cells, but this was not obvious in controls (Figure 3). These results indicated that human prorenin molecules entered the urinary space where they were taken up by renal 

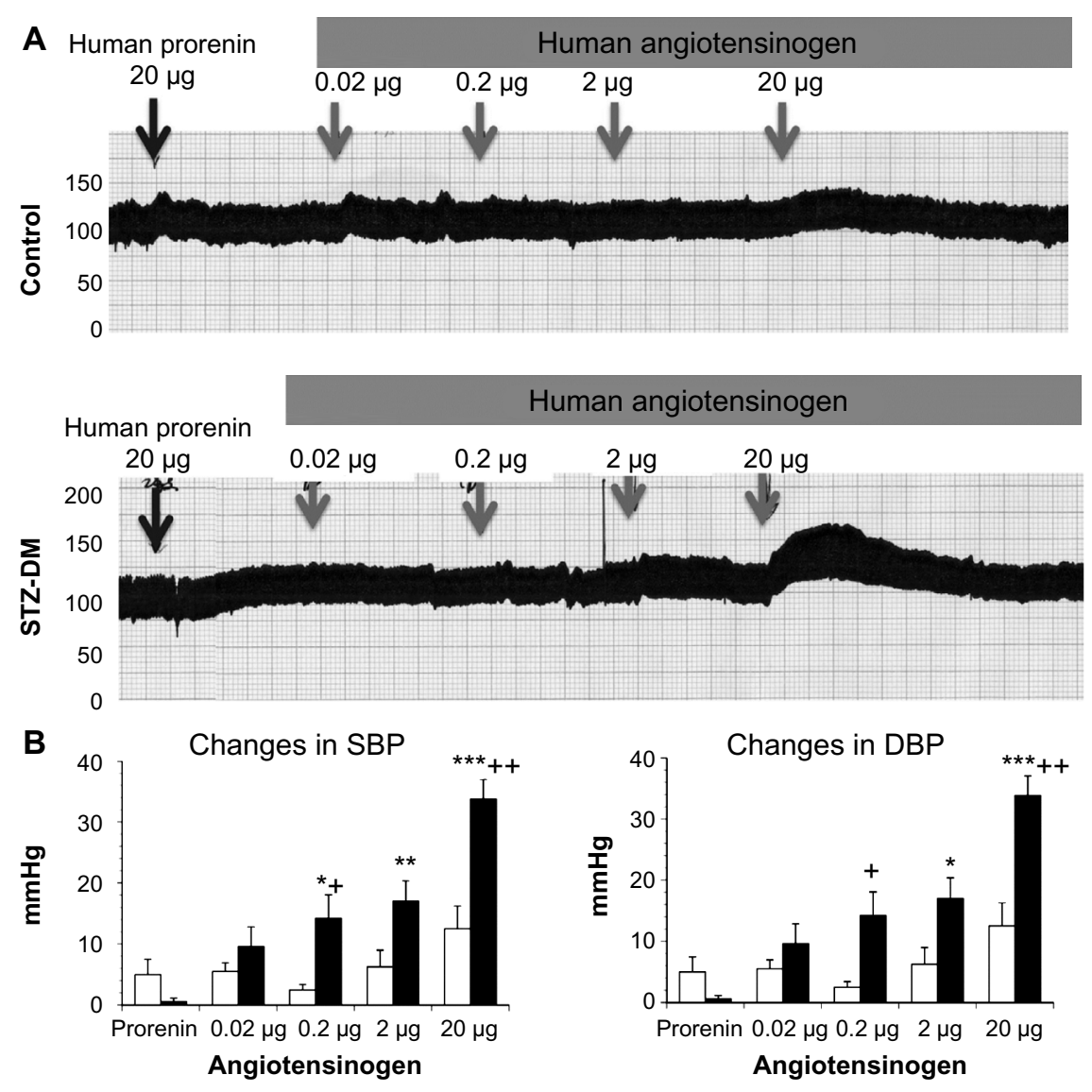

Figure I Changes in the blood pressure of control and STZ-induced DM rats after the administration of human prorenin and human angiotensinogen.

Notes: (A) Records of arterial blood pressure in a control and diabetic rat injected with human prorenin and angiotensinogen. (B) Graphs showing the variations in the SBP and DBP in control and diabetic rats. A significant increase in blood pressure was observed only in the diabetic rats. White bars indicate control and black bars indicate diabetic rats. ${ }^{* P}<0.05, * * P<0.01$, ${ }^{*} * P<0.001$ vs changes after prorenin injection for each group, ${ }^{+P}<0.05,{ }^{++} \mathrm{P}<0.01$ vs the control rats at the same dose. $\mathrm{N}=4$ in each group.

Abbreviations: DBP, diastolic blood pressure; DM, diabetes mellitus; SBP, systolic blood pressure; STZ, streptozotocin.

cells, especially podocytes and tubular epithelial cells. This uptake was enhanced in DM rats.

\section{Enhanced endocytosis of prorenin mediated by the PRR and megalin in diabetic rats}

Megalin and PRR are expressed by podocytes and renal tubular epithelial cells. An immunoprecipitation analysis identified PRR in podocytes and cells of the macula densa and CCDs, and, to a lesser extent, of the proximal tubules. However, the proximal tubular immunoreactivity appeared to be increased in DM rats (Figure 4A-D). A Western blot analysis for PRR in kidney homogenates confirmed that the renal PRR expression was increased almost threefold in DM rats (Figure 4E and F). An immunoprecipitation study of human prorenin using antibodies for megalin or the PRR demonstrated that human prorenin was bound to both megalin and the PRR. The amount of bound prorenin was increased in DM rats (Figure 5). Thus, endocytic uptake of human prorenin by the kidney may be mediated by both the PRR and megalin, both of which were enhanced in DM rats.

\section{Uptake of human angiotensinogen}

Following its IV injection, immunoreactive human angiotensinogen was detected in podocytes, proximal tubules, macula densa, and collecting ducts, which was a similar distribution to that found for human prorenin and the PRR. The immunoreactive staining for angiotensinogen appeared increased in DM rats, notably in the podocytes (Figure 6A and $\mathrm{B}$ ) and the vascular endothelial cells (Figure 6C and D). Indeed, there was an increase in $52 \mathrm{kDa}$ human angiotensinogen detected by a Western blot of glomerular fraction isolated from DM rats, whereas it was a slight increase in the tubular fraction (Figure 6E-G). The specificity of the antibody was confirmed by Western blot demonstrating a reaction with human angiotensinogen in a dose-dependent manner, but faintly with rat angiotensinogen (Figure 6E). 


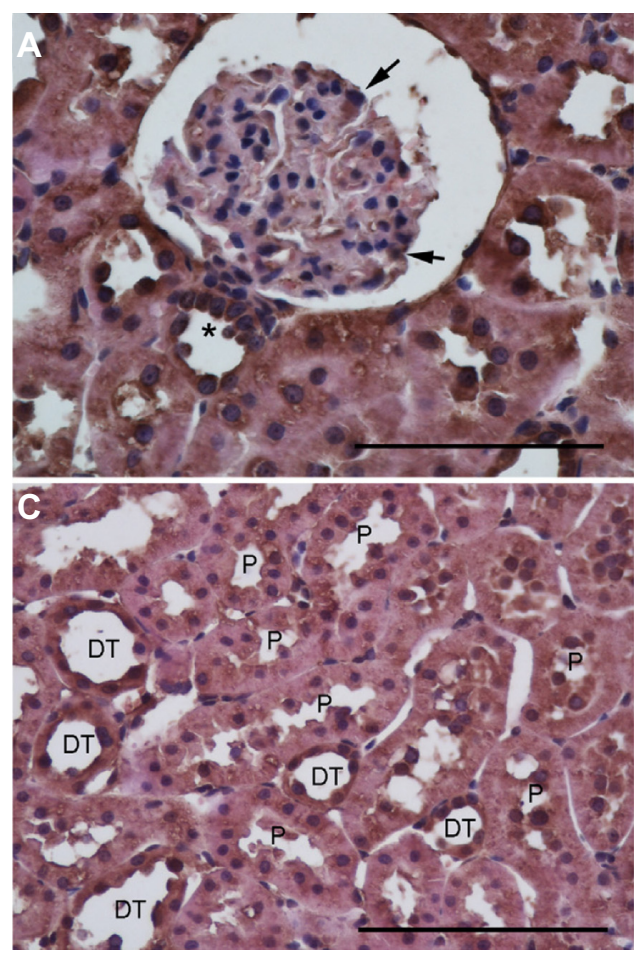

Control

E

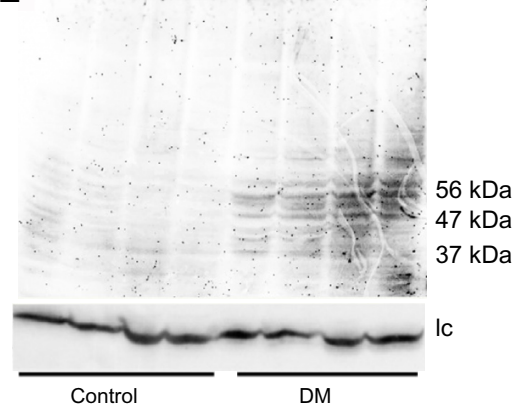

$\mathbf{F}$

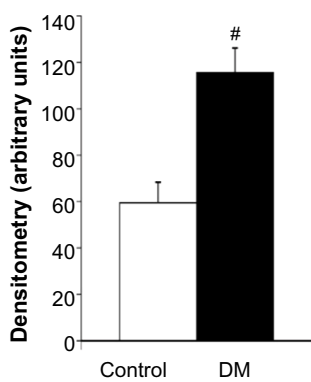

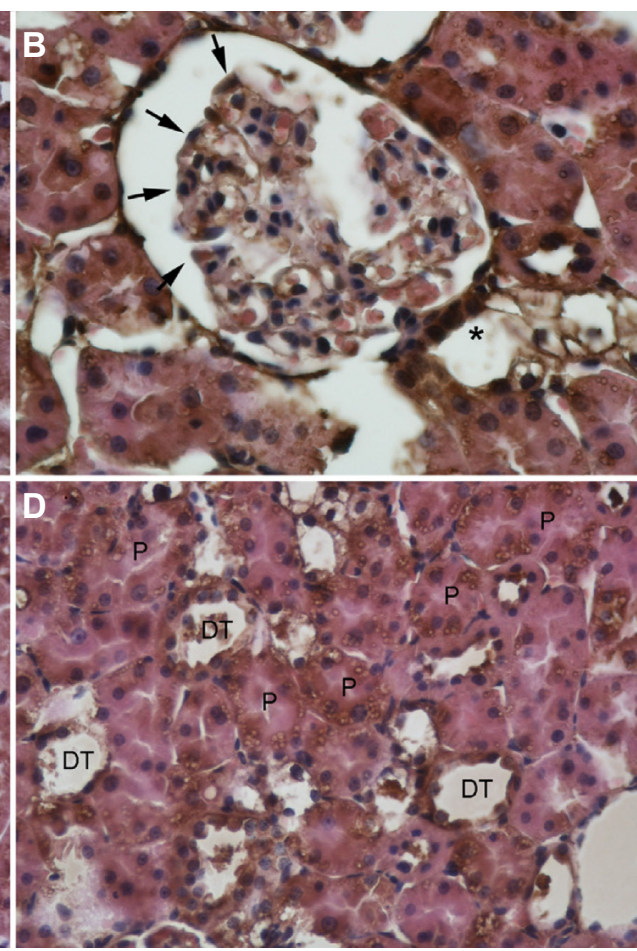

DM

G

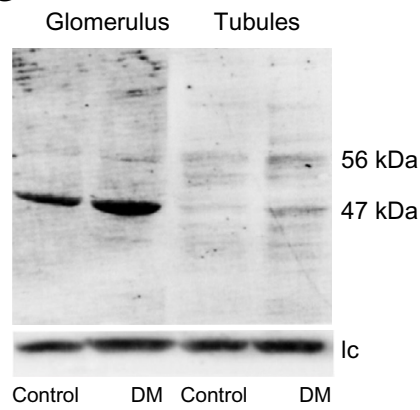

Figure 2 The uptake of exogenous human prorenin is enhanced in diabetic rats.

Notes: Immunostaining for human prorenin (brown color staining in A-D) in podocytes (arrows), proximal tubules (P), macula densa (asterisks), and distal nephrons (DT) including cortical collecting ducts, appeared enhanced in diabetic rats (B and $\mathbf{D})$ compared with controls $(\mathbf{A}$ and $\mathbf{C})$. Bar: $100 \mu \mathrm{m}$. A Western blot analysis for human prorenin demonstrating an increased uptake of human prorenin in the whole kidney homogenates $(\mathbf{E}$ and $\mathbf{F})$ and also in glomerular and tubular fractions in diabetic rats (G). ${ }^{\#}<<0.0 \mathbf{I}$ vs control. Abbreviations: DM, streptozotocin-induced diabetes mellitus rats; Ic, loading control.

\section{Renal Ang II concentration and ACE type I expression}

Renal concentrations of Ang II were not significantly different between DM and control rats (6.1 \pm 1.6 in DM vs $5.7 \pm 1.0 \mathrm{pg} / \mathrm{mg}$ protein in control, ns). An immunohistochemical study detected ACE type 1 (ACE-1; Figure 7) mainly in the proximal tubules, especially in the brush border membranes of control kidneys. Its expression in DM rats was absent in proximal tubules but increased in glomeruli.

\section{Discussion}

The main new findings in this study are that exogenous human prorenin and human angiotensinogen were filtrated and taken up by renal cells, including podocytes, proximal tubular cells, macula densa cells, and CCD cells. This process was enhanced in diabetic rats. Renal uptake was mediated by binding to the PRR and megalin.

The origin of renal angiotensinogen remains controversial. The findings of enhanced mRNA for angiotensinogen in the proximal tubule cells of diabetic rats ${ }^{25,26}$ certainly suggested that it originated predominantly from the proximal tubule. ${ }^{27,28}$ This is important since renal angiotensinogen excretion has been used as a marker of intrarenal Ang II and early kidney injury in conditions that include DM.,29 However, there have been conflicting conclusions concerning the origin of renal angiotensinogen and the role of 

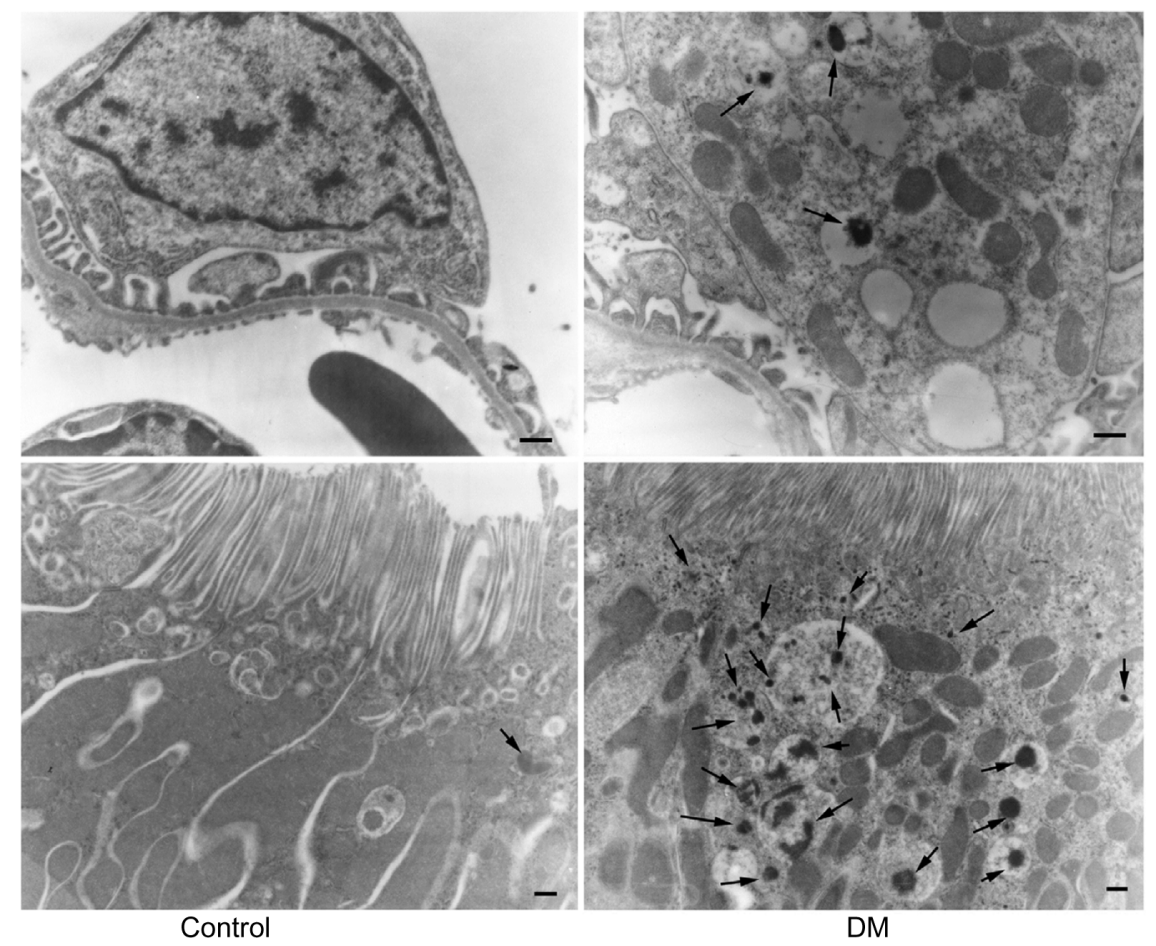

Figure 3 Pre-embedding electron microscopy for human prorenin.

Notes: Black label for human prorenin was detected in podocyte vesicles and proximal tubules in diabetic rats 40 minutes after its first intravenous injection (arrows), but was barely detected in controls. Bar: $500 \mathrm{~nm}$.

Abbreviation: DM, diabetes mellitus rats.

renal tubular angiotensinogen synthesis. ${ }^{16,17,30-32}$ Thus, Pohl et $\mathrm{al}^{16}$ reported abundant endocytosis of angiotensinogen by megalin in the $\mathrm{S} 1$ segment of proximal tubules of mice, whereas angiotensinogen mRNA was localized to the S2/ S3 segments where angiotensinogen protein expression was low. Furthermore, inhibition of endocytosis by colchicine decreased renal angiotensinogen levels. ${ }^{33}$ Matsusaka et $\mathrm{al}^{17}$ reported that angiotensinogen was expressed in the kidneys of kidney-specific angiotensinogen gene knockout mice but was absent from the kidneys of liver-specific angiotensinogen knockout mice. They concluded that renal angiotensinogen derives predominantly from angiotensinogen secreted into the bloodstream by the liver and extracted by the kidneys. ${ }^{17}$

Our study is the first to show directly that exogenous angiotensinogen is taken up in the kidney preferentially in diabetic rats with microalbuminuria. This is consistent with increased glomerular filtration of angiotensinogen since its excretion parallels that of albumin. ${ }^{27,28}$ Indeed, the estimated glomerular sieving coefficients of prorenin and angiotensinogen, judged from their molecular size, are approximately tenfold higher than that of albumin..$^{34,35}$ Moreover, the injected human angiotensinogen was detected in glomerular podocytes and proximal tubules and its uptake into glomerular podocytes was increased in diabetic rats, suggesting increased filtration of angiotensinogen associated with albuminuria.

A second important finding from our study was that circulating human prorenin also was taken up in excess by diabetic kidneys and was localized to the podocytes, proximal tubules, and distal nephron, including the CCD where PRR is expressed. Immunoprecipitation studies indicated that prorenin was bound to, and taken up by both PRR and megalin consistent with prior reports that prorenin uptake and activation occurs in vascular endothelial cells. ${ }^{36,37}$ Thus, an enhanced uptake of filtered angiotensinogen and prorenin by podocytes and renal tubules contributed to an enhanced RAS in diabetic kidneys.

ACE-1 expression was reduced in the brush border membrane of the proximal tubule of diabetic rats, but was increased in glomeruli and vascular endothelial cells, consistent with previous reports. ${ }^{5,38} \mathrm{~A}$ limited expression of renal tubular ACE-1 may underlie the maintained renal Ang II in DM rats despite enhanced uptake of prorenin and angiotensinogen. There are conflicting reports of renal Ang II levels in diabetes, ${ }^{7,11,39}$ which may reflect the degree of filtration of angiotensinogen as well as salt intake. An increased uptake of exogenous angiotensinogen 

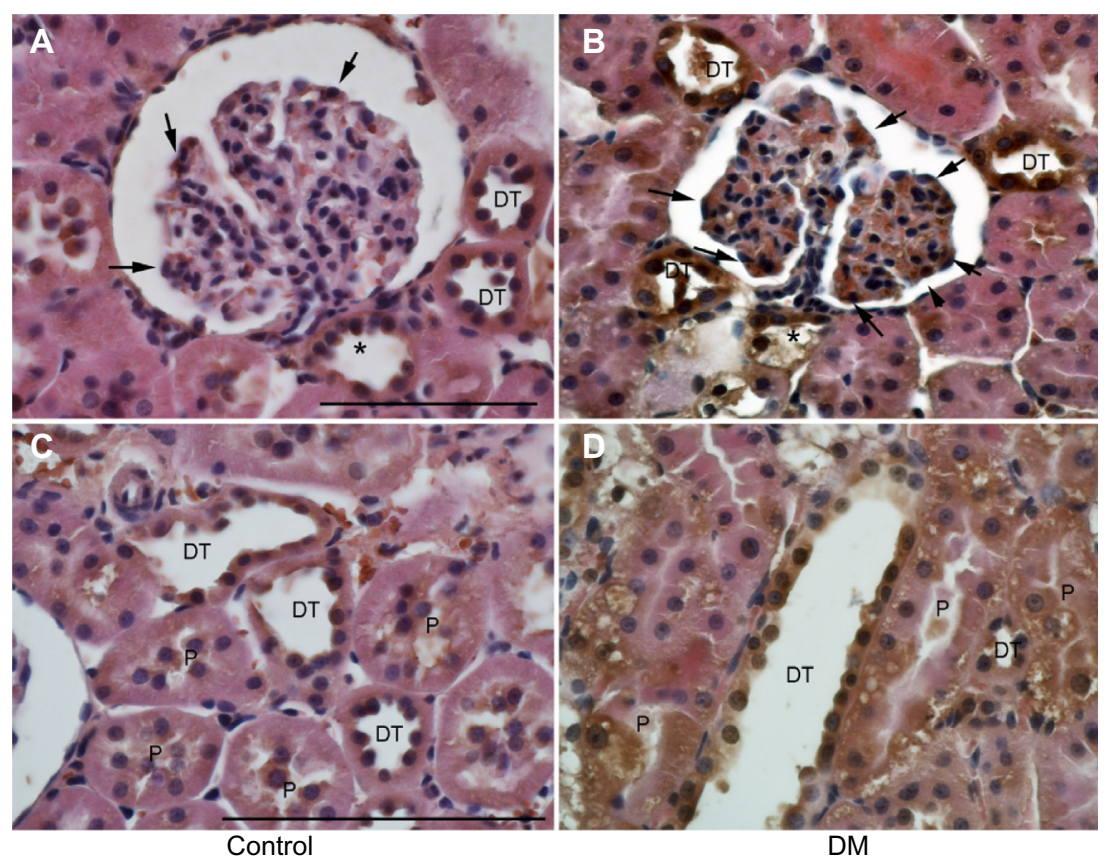

$\mathbf{E}$
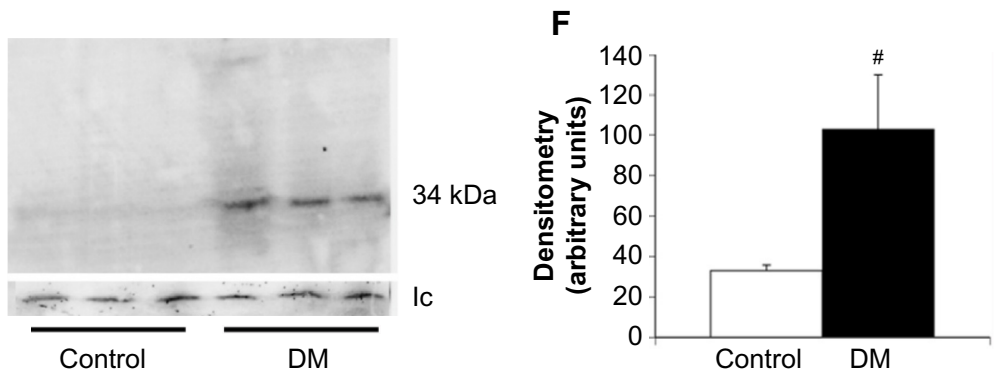

Figure 4 The expression of the prorenin receptor in normal and diabetic rats.

Notes: Immunohistochemical analysis of the prorenin receptor in podocytes (arrows), proximal tubules (P), macula densa (asterisks), and distal nephron including cortical collecting ducts (DT), appeared increased in diabetic rats (B and $\mathbf{D})$ compared with controls ( $\mathbf{A}$ and $\mathbf{C})$. This was confirmed by the results of a Western blot analysis (E and F). $" P<0.05$ vs control. Bar: $100 \mu \mathrm{m}$.

Abbreviations: DM, diabetes mellitus rats; Ic, loading control.

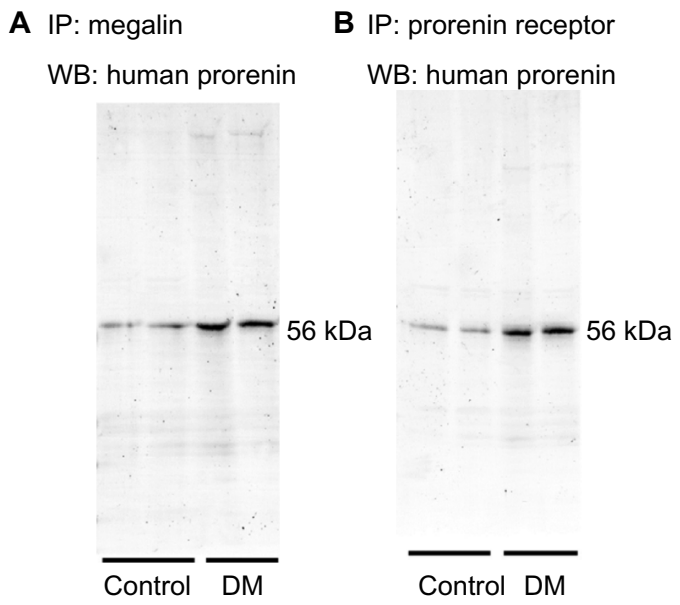

Figure 5 Immunoprecipitation of human prorenin by antibodies for megalin (A) or the prorenin receptor $(\mathbf{B})$.

Note: The level of renal human prorenin bound to both megalin and the prorenin receptor was increased in diabetic rats compared with controls.

Abbreviations: DM, diabetes mellitus rats; IP, immunoprecipitation; WB, Western blot. by the vascular endothelial cells in DM rats may have contributed to the enhanced BP responses of these rats to injected human angiotensinogen and to hypertension in DM.

The enhanced renal uptake of prorenin evident in podocyte, proximal tubule, macula densa, and CCDs of DM rats mediated as an up-regulated PRR at their sites could have two consequences. First, increased circulating prorenin in $\mathrm{DM}^{40}$ combined with increased filtration in proteinuric states may enhance tubular renin activity since binding of prorenin to the PRR generates active renin. Second, the PRR activates the mitogen-activated protein kinase-extracellular signalregulated kinase $1 / 2$ pathway that increases the expression of profibrotic molecules such as transforming growth factor $\beta 1$, plasminogen activator inhibitor-1, fibronectin, and collagen, and also induces mesangial proliferation. ${ }^{41-43}$ Thus, an enhanced plasma level and filtration of prorenin combined 


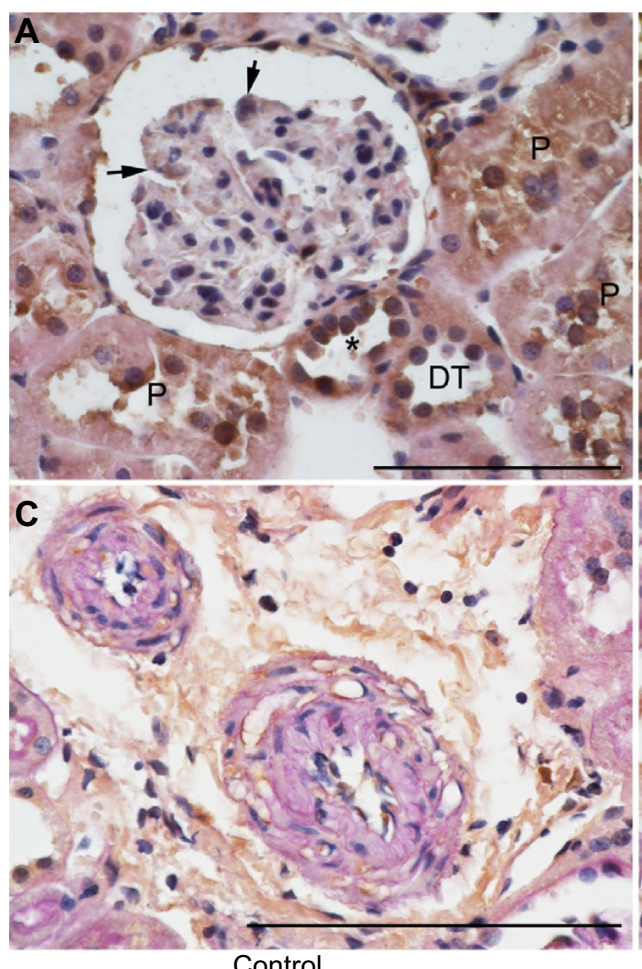

Control

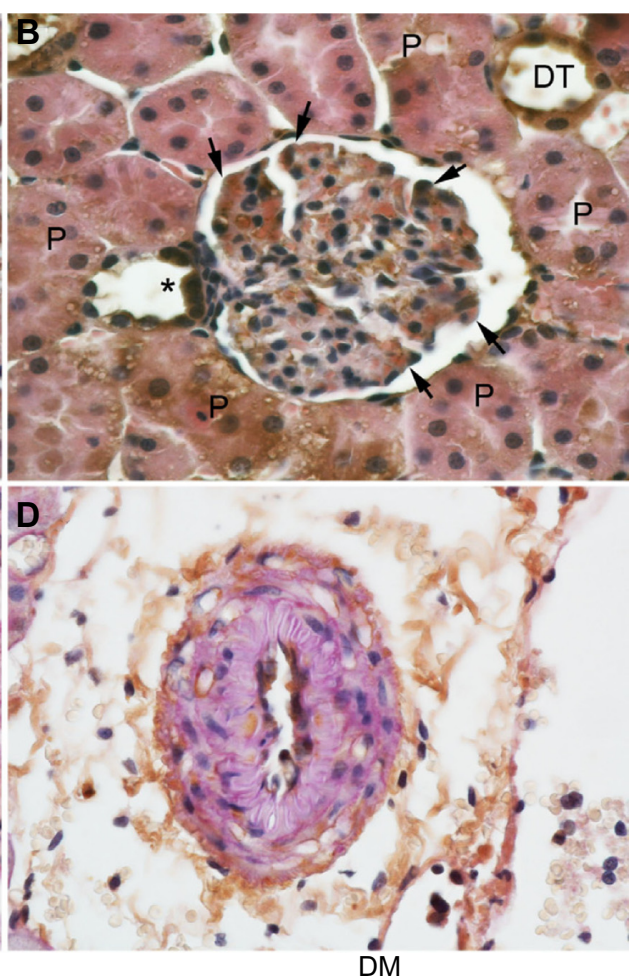

DM E Human Atg (ng) Rat Atg (ng)
$101001,000101001,000$
F Glomerulus Tubules

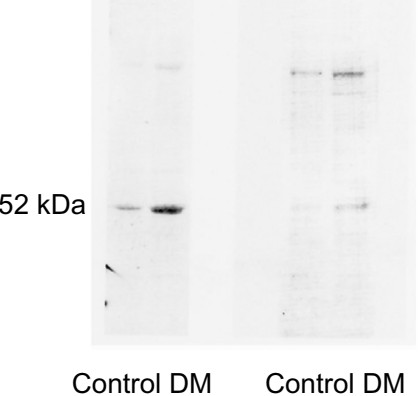

G Glomerulus Tubules

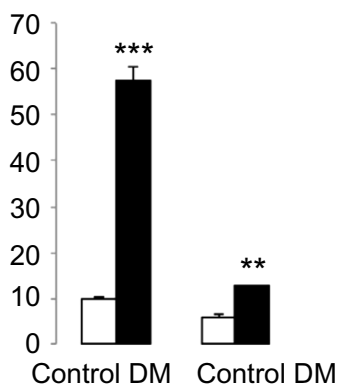

Figure 6 Human angiotensinogen uptake in normal and diabetic rats.

Notes: Immunohistochemical analysis for human angiotensinogen expression (brown color staining in A-D) in podocytes (arrows), proximal tubules (P), *macula densa and the distal nephron (DT) including collecting ducts and endothelial cells of renal small artery (C and D) appeared increased in diabetic rats, especially in podocytes. Western blot for human angiotensinogen demonstrated that the specificity of the antibody which reacted with human angiotensinogen in a dose-dependent manner, but only weakly with rat angiotensinogen (E). Western blot analysis showed increased human angiotensinogen uptake in the glomerular fraction of DM rats, but slightly increased in the tubular fraction of DM rats compared with those of control (F and $\mathbf{G})$. $* * P<0.00$ I, $* * * P<0.000$ I vs control. Bar: $100 \mu \mathrm{m}$.

Abbreviations: Atg, angiotensinogen; DM, diabetes mellitus rats.

with an enhanced expression of the PRR may promote renal damage in DM, and PRR blockade was shown to improve DM nephropathy. ${ }^{44}$

Although microalbuminuria is an established risk factor for renal disease progression and cardiovascular disease, the mechanism is unknown. ${ }^{45,46}$ Approximately $3 \mathrm{~g}$ of albumin are filtered daily by the glomeruli and reabsorbed by the tubules. ${ }^{34}$ Albumin endocytosis is enhanced in the podocytes and proximal tubules in proteinuric conditions. ${ }^{47}$ We now demonstrate enhanced endocytosis of prorenin and angiotensinogen in the kidneys and the vascular endothelium of DM rats with albuminuria. Thus, the true culprit molecules that mediate progression of CKD and the development of CVD during microalbuminuria may be angiotensinogen and prorenin whose glomerular filtration, tubular uptake, and renal excretion is proportional to microalbuminuria. ${ }^{17,27,28,30}$ This scenario would identify albumin excretion as a marker for renal filtration and uptake of these RAS components.

\section{Conclusion}

Endocytosis of exogenous prorenin and/or angiotensinogen molecules was enhanced in the podocytes, renal tubules, and vascular endothelial cells in diabetic rats, implying that the glomerular filtration and uptake of these molecules by 


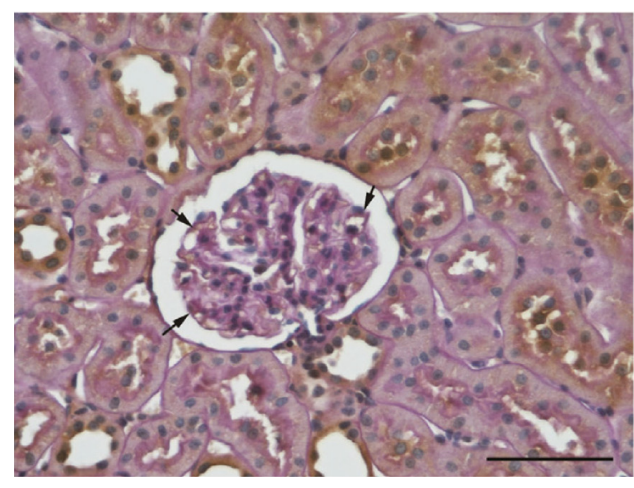

Control

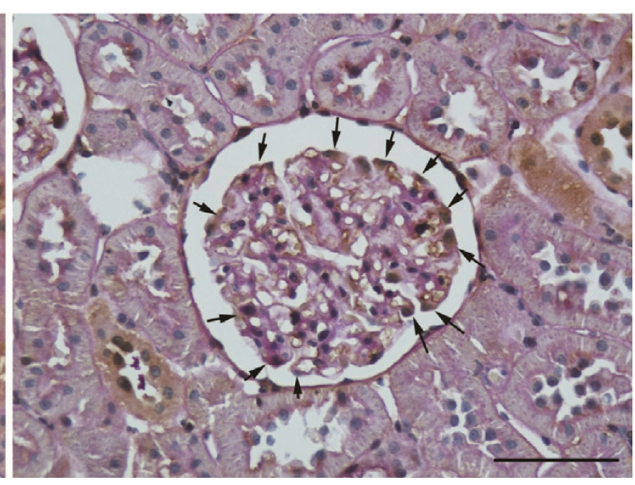

$\mathrm{DM}$

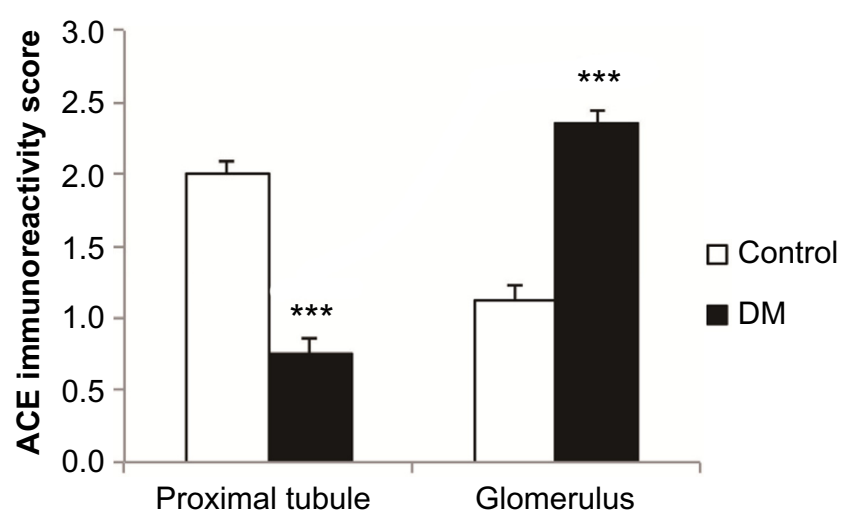

Figure 7 The expression of ACE in normal and diabetic rats.

Notes: The semiquantification of immunoreactivity for ACE indicated reduced brush border membrane of proximal tubules but enhanced podocytes of diabetic rats (arrows). $* * * P<0.000$ I. Bar: $100 \mu \mathrm{m}$.

Abbreviations: $\mathrm{ACE}$, angiotensin-converting enzyme; DM, diabetes mellitus rats.

renal cells and the blood vessels may activate a local RAS in diabetes.

\section{Acknowledgments}

This work was partly supported by a grant-in-aid for scientific research from Japan Science Promotion Foundation to AT (C-23591214) and by grants from the NIH to CSW (DK-049870; DK-036079; HL-68686).

\section{Author contributions}

AT and SK performed animal studies and all authors contributed toward data analysis, drafting and critically revising the paper and agree to be accountable for all aspects of the work.

\section{Disclosure}

The authors report no conflicts of interest in this work.

\section{References}

1. Bader M. Tissue renin-angiotensin-aldosterone systems: targets for pharmacological therapy. Annu Rev Pharmacol Toxicol. 2010;50:439-465.

2. Paul M, Poyan Mehr A, Kreutz R. Physiology of local renin-angiotensin systems. Physiol Rev. 2006;86(3):747-803.
3. Carey RM, Siragy HM. The intrarenal renin-angiotensin system and diabetic nephropathy. Trends Endocrinol Metab. 2003;14(6):274-281.

4. Siragy HM, Carey RM. Role of the intrarenal renin-angiotensin-aldosterone system in chronic kidney disease. Am J Nephrol. 2010;31(6): 541-550.

5. Anderson S, Jung FF, Ingelfinger JR. Renal renin-angiotensin system in diabetes: functional, immunohistochemical, and molecular biological correlations. Am J Physiol. 1993;265(4 Pt 2):F477-F486.

6. Price DA, Porter LE, Gordon M, et al. The paradox of the low-renin state in diabetic nephropathy. J Am Soc Nephrol. 1999;10(11):2382-2391.

7. Vallon V, Wead LM, Blantz RC. Renal hemodynamics and plasma and kidney angiotensin II in established diabetes mellitus in rats: effect of sodium and salt restriction. JAm Soc Nephrol. 1995;5(10):1761-1767.

8. Kobori H, Nangaku M, Navar LG, Nishiyama A. The intrarenal reninangiotensin system: from physiology to the pathobiology of hypertension and kidney disease. Pharmacol Rev. 2007;59(3):251-287.

9. Nagai Y, Yao L, Kobori H, et al. Temporary angiotensin II blockade at the prediabetic stage attenuates the development of renal injury in type 2 diabetic rats. J Am Soc Nephrol. 2005;16(3):703-711.

10. Siragy HM, Awad A, Abadir P, Webb R. The angiotensin II type 1 receptor mediates renal interstitial content of tumor necrosis factor-alpha in diabetic rats. Endocrinology. 2003;144(6):2229-2233.

11. Zimpelmann J, Kumar D, Levine DZ, et al. Early diabetes mellitus stimulates proximal tubule renin mRNA expression in the rat. Kidney Int. 2000;58(6):2320-2330.

12. Peti-Peterdi J, Fintha A, Fuson AL, Tousson A, Chow RH. Realtime imaging of renin release in vitro. Am J Physiol Renal Physiol. 2004;287(2):F329-F335.

13. Krop M, Danser AH. Circulating versus tissue renin-angiotensin system: on the origin of (pro)renin. Curr Hypertens Rep. 2008;10(2):112-118. 
14. Braam B, Mitchell KD, Fox J, Navar LG. Proximal tubular secretion of angiotensin II in rats. Am J Physiol. 1993;264(5 Pt 2):F891-F898.

15. Navar LG, Harrison-Bernard LM, Nishiyama A, Kobori H. Regulation of intrarenal angiotensin II in hypertension. Hypertension. 2002;39(2 Pt 2): 316-322.

16. Pohl M, Kaminski H, Castrop H, et al. Intrarenal renin angiotensin system revisited: role of megalin-dependent endocytosis along the proximal nephron. J Biol Chem. 2010;285(53):41935-41946.

17. Matsusaka T, Niimura F, Shimizu A, et al. Liver angiotensinogen is the primary source of renal angiotensin II. J Am Soc Nephrol. 2012;23(7): $1181-1189$.

18. Nguyen G, Danser AH. Prorenin and (pro)renin receptor: a review of available data from in vitro studies and experimental models in rodents. Exp Physiol. 2008;93(5):557-563.

19. Onozato ML, Tojo A, Leiper J, Fujita T, Palm F, Wilcox CS. Expression of NG,NG-dimethylarginine dimethylaminohydrolase and protein arginine N-methyltransferase isoforms in diabetic rat kidney: effects of angiotensin II receptor blockers. Diabetes. 2008;57(1):172-180.

20. Tojo A, Onozato ML, Kobayashi N, Goto A, Matsuoka H, Fujita T. Angiotensin II and oxidative stress in Dahl Salt-sensitive rat with heart failure. Hypertension. 2002;40(6):834-839.

21. Onozato ML, Tojo A, Goto A, Fujita T, Wilcox CS. Oxidative stress and nitric oxide synthase in rat diabetic nephropathy: effects of ACEI and ARB. Kidney Int. 2002;61(1):186-194.

22. Tojo A, Onozato ML, Kitiyakara C, et al. Glomerular albumin filtration through podocyte cell body in puromycin aminonucleoside nephrotic rat. Med Mol Morphol. 2008;41(2):92-98.

23. Tojo A, Welch WJ, Bremer V, et al. Colocalization of demethylating enzymes and NOS and functional effects of methylarginines in rat kidney. Kidney Int. 1997;52(6):1593-1601.

24. Ookata K, Tojo A, Suzuki Y, et al. Localization of inward rectifier potassium channel Kir7.1 in the basolateral membrane of distal nephron and collecting duct. JAm Soc Nephrol. 2000;11(11):1987-1994.

25. Hsieh TJ, Zhang SL, Filep JG, Tang SS, Ingelfinger JR, Chan JS. High glucose stimulates angiotensinogen gene expression via reactive oxygen species generation in rat kidney proximal tubular cells. Endocrinology. 2002;143(8):2975-2985.

26. Zhang SL, Filep JG, Hohman TC, Tang SS, Ingelfinger JR, Chan JS. Molecular mechanisms of glucose action on angiotensinogen gene expression in rat proximal tubular cells. Kidney Int. 1999;55(2):454-464.

27. Kobori H, Ohashi N, Katsurada A, et al. Urinary angiotensinogen as a potential biomarker of severity of chronic kidney diseases. J Am Soc Hypertens. 2008;2(5):349-354.

28. van den Heuvel M, Batenburg WW, Jainandunsing S, et al. Urinary renin, but not angiotensinogen or aldosterone, reflects the renal reninangiotensin-aldosterone system activity and the efficacy of reninangiotensin-aldosterone system blockade in the kidney. J Hypertens. 2011;29(11):2147-2155.

29. Nakano D, Kobori H, Burford JL, et al. Multiphoton imaging of the glomerular permeability of angiotensinogen. J Am Soc Nephrol. 2012;23(11):1847-1856.

30. Kobori H, Nishiyama A, Harrison-Bernard LM, Navar LG. Urinary angiotensinogen as an indicator of intrarenal angiotensin status in hypertension. Hypertension. 2003;41(1):42-49.
31. Rohrwasser A, Morgan T, Dillon HF, et al. Elements of a paracrine tubular renin-angiotensin system along the entire nephron. Hypertension. 1999;34(6):1265-1274.

32. Gociman B, Rohrwasser A, Lantelme P, et al. Expression of angiotensinogen in proximal tubule as a function of glomerular filtration rate. Kidney Int. 2004;65(6):2153-2160.

33. Richoux JP, Cordonnier JL, Bouhnik J, et al. Immunocytochemical localization of angiotensinogen in rat liver and kidney. Cell Tissue Res. 1983;233(2):439-451.

34. Tojo A, Endou $\mathrm{H}$. Intrarenal handling of proteins in rats using fractional micropuncture technique. Am J Physiol. 1992;263(4 Pt 2):F601-F606.

35. Norden AG, Lapsley M, Lee PJ, et al. Glomerular protein sieving and implications for renal failure in Fanconi syndrome. Kidney Int. 2001;60(5):1885-1892.

36. Admiraal PJ, van Kesteren CA, Danser AH, Derkx FH, Sluiter W, Schalekamp MA. Uptake and proteolytic activation of prorenin by cultured human endothelial cells. J Hypertens. 1999;17(5):621-629.

37. van den Eijnden MM, Saris JJ, de Bruin RJ, et al. Prorenin accumulation and activation in human endothelial cells: importance of mannose 6-phosphate receptors. Arterioscler Thromb Vasc Biol. 2001;21(6): 911-916.

38. Mizuiri S, Yoshikawa H, Tanegashima M, et al. Renal ACE immunohistochemical localization in NIDDM patients with nephropathy. Am J Kidney Dis. 1998;31(2):301-307.

39. Mezzano S, Droguett A, Burgos ME, et al. Renin-angiotensin system activation and interstitial inflammation in human diabetic nephropathy. Kidney Int Suppl. 2003;86:S64-S70.

40. Deinum J, Ronn B, Mathiesen E, Derkx FH, Hop WC, Schalekamp MA. Increase in serum prorenin precedes onset of microalbuminuria in patients with insulin-dependent diabetes mellitus. Diabetologia. 1999;42(8):1006-1010.

41. Nguyen G, Delarue F, Burckle C, Bouzhir L, Giller T, Sraer JD. Pivotal role of the renin/prorenin receptor in angiotensin II production and cellular responses to renin. J Clin Invest. 2002;109(11):1417-1427.

42. Huang Y, Wongamorntham S, Kasting J, et al. Renin increases mesangial cell transforming growth factor-beta 1 and matrix proteins through receptor-mediated, angiotensin II-independent mechanisms. Kidney Int. 2006;69(1):105-113.

43. Feldt S, Batenburg WW, Mazak I, et al. Prorenin and renin-induced extracellular signal-regulated kinase $1 / 2$ activation in monocytes is not blocked by aliskiren or the handle-region peptide. Hypertension. 2008;51(3):682-688.

44. Ichihara A, Suzuki F, Nakagawa T, et al. Prorenin receptor blockade inhibits development of glomerulosclerosis in diabetic angiotensin II type 1a receptor-deficient mice. J Am Soc Nephrol. 2006;17(7):1950-1961.

45. Wachtell $\mathrm{K}$, Ibsen $\mathrm{H}$, Olsen $\mathrm{MH}$, et al. Albuminuria and cardiovascular risk in hypertensive patients with left ventricular hypertrophy: the LIFE study. Ann Intern Med. 2003;139(11):901-906.

46. Hillege HL, Fidler V, Diercks GF, et al. Urinary albumin excretion predicts cardiovascular and noncardiovascular mortality in general population. Circulation. 2002;106(14):1777-1782.

47. Tojo A. The role of the kidney in protein metabolism: the capacity of tubular lysosomal proteolysis in nephrotic syndrome. Kidney Int. 2013; 84(5):861-863.

Diabetes, Metabolic Syndrome and Obesity: Targets and Therapy

Dovepress

\section{Publish your work in this journal}

Diabetes, Metabolic Syndrome and Obesity: Targets and Therapy is an international, peer-reviewed open-access journal committed to the rapid publication of the latest laboratory and clinical findings in the fields of diabetes, metabolic syndrome and obesity research. Original research, review, case reports, hypothesis formation, expert opinion and commentaries are all considered for publication. The manuscript management system is completely online and includes a very quick and fair peer-review system, which is all easy to use. Visit http://www.dovepress.com/testimonials.php to read real quotes from published authors. 